\title{
A MULTIFRACTAL WAVELET MODEL FOR POSITIVE PROCESSES
}

\author{
Matthew S. Crouse, Rudolf H. Riedi, Vinay J. Ribeiro, and Richard G. Baraniuk \\ Department of Electrical and Computer Engineering, \\ Rice University, Houston, TX 77005
}

\begin{abstract}
In this paper, we describe a new multiscale model for characterizing positive-valued and long-range dependent data. The model uses the Haar wavelet transform and puts a constraint on the wavelet coefficients to guarantee positivity, which results in a swift $O(N)$ algorithm to synthesize $N$-point data sets. We elucidate our model's ability to capture the covariance structure of real data, study its multifractal properties, and derive a scheme for matching it to real data observations. We demonstrate the model's utility by applying it to network traffic synthesis. The flexibility and accuracy of the model and fitting procedure result in a close match to the real data statistics (variance-time plots) and queuing behavior.
\end{abstract}

\section{INTRODUCTION}

Fractals models arise frequently in a variety of scientific disciplines, such as physics, chemistry, astronomy, and biology. In DSP, fractals have long proven useful for applications such as computer graphics and texture modeling [1]. More recently, fractal models have had a major impact on the analysis of data communication networks such as the Internet. In their landmark paper [2], Leland et al. demonstrated that network traffic exhibits fractal properties such as self-similarity, "burstiness," and long-range dependence (LRD) that are inadequately described by classical traffic models. Characterization of these fractal properties, particularly LRD, has provided exciting new insights into network behavior and performance.

Fractals are geometric objects that exhibit an irregular structure at all resolutions. Most fractals are self-similar; if we "zoom" (in or out) of the fractal, we obtain a picture similar to the original. Deterministic fractals usually have a highly specific structure that can be constructed through a few simple steps. Real-world phenomena can rarely be described using such simple models. Nevertheless, "similarity on all scales" can hold in a statistical sense, leading to the notion of random fractals.

As the pre-eminent random fractal model, fractional Brownian motion ( $\mathrm{fBm}$ ) has played a central rôle in many fields $[2,3]$. FBm is the unique Gaussian process with stationary increments and the following scaling property for all $a>0$

$$
B(a t) \stackrel{f d}{=} a^{H} B(t)
$$

with the equality in (finite-dimensional) distribution. The parameter $H, 0<H<1$, is known as the Hurst parameter. It rules the LRD of $\mathrm{fBm}$, as we will see later, but it also governs its local "spikiness." In particular, for all $t$

$$
B(t+s)-B(t) \simeq s^{H},
$$

This work was supported by the National Science Foundation, grant no. MIP-9457438, and by DARPA/AFOSR, grant no. F49620-97-1-0513. URL: www.dsp.rice.edu. Email:\{mcrouse, riedi, vinay, richb\} @rice.edu. meaning that, for $0<H<1, \mathrm{fBm}$ has "infinite slope" everywhere.

The statistical self-similarity (1) of $\mathrm{fBm}$ has proven most useful for signal modeling, since it efficiently captures signal features such as burstiness and LRD, while still allowing tractable theoretical analysis [2]. Nevertheless, models based on $\mathrm{fBm}$ can be too restrictive to adequately characterize many types of signals.

First, strictly self-similar scaling behavior is not always realistic. For example, $H$ in (1) may vary when measured over different values of $a$, or $H$ in (2) may depend on $t$. Second, fBm models are inherently Gaussian. Many signals have positive increments and, hence, are non-Gaussian.

For applications such as network traffic $[4,5]$ and turbulence analysis [6], the statistics of such signals can be more accurately characterized using a multifractal analysis, which describes how the signal's scaling behavior varies across the signal. In this paper, we develop a multifractal signal model especially suited to positive-valued data with LRD. Modeling of such data is not only vital for networking $[2,4]$, but it also provides key insights into a host of other applications such as turbulence and geophysics [6].

We call our model the multifractal wavelet model (MWM) because of its wavelet-domain formulation. With its simple Haarwavelet construction, the MWM is simple to apply, yet it can characterize LRD and a number of different multifractal properties. Since we cannot treat the MWM in full detail in this paper, we refer the interested reader to [7] for a more in-depth treatment.

\section{FBM AND LRD}

Although we analyze $\mathrm{fBm}$ from a continuous-time point of view, for practical computations and simulations, we often work with sampled continuous-time $\mathrm{fBm}$. The increments process of sampled fBm

$$
X[n]:=B(n)-B(n-1)
$$

defines a stationary Gaussian sequence known as discrete fractional Gaussian noise (fGn) with covariance behavior [1]

$$
r_{X}[k] \simeq|k|^{2 H-2}, \text { for }|k| \text { large. }
$$

For $1 / 2<H<1$, the covariance of fGn is strictly positive and decays so slowly that it is non-summable (i.e., $\sum_{k} r_{X}[k]=$ $\infty$ ). This non-summability, corresponding to positive, slowlydecaying covariances over large time lags, defines LRD.

The LRD of fGn can be equivalently characterized in terms of how the aggregated processes

$$
X^{(m)}(n):=\frac{1}{m} \sum_{i=(k-1) m+1}^{k m} X(i)
$$

behave. It follows from (1) that $X(n) \stackrel{f d}{=} m^{1-H} X^{(m)}(n)$. 
(a)

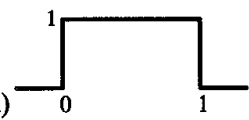

(b)

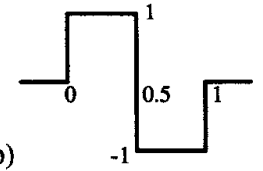

Figure 1: (a) Haar scaling function $\phi$ and (b) Haar wavelet function $\psi$

Hence, a $\log -\log$ plot of the variance of $X^{(m)}(n)$ as a function of $m-$ known as a variance-time plot - will have a slope of $2 H-2$. The variance-time plot can characterize LRD in nonGaussian, non-zero-mean data as well [2].

\section{WAVELETS AND LRD}

The discrete wavelet transform is a multi-scale signal representation of the form [8]

$$
\begin{aligned}
x(t)= & \sum_{k} u_{k} 2^{-J_{0} / 2} \phi\left(2^{-J_{0}} t-k\right)+ \\
& \sum_{j=-\infty}^{J_{0}} \sum_{k} w_{j, k} 2^{-j / 2} \psi\left(2^{-j} t-k\right), \quad j, k \in \mathbb{Z}
\end{aligned}
$$

with $J_{0}$ the coarsest scale and $u_{k}$ and $w_{j, k}$ the scaling and wavelet coefficients, respectively. The scaling coefficients may be viewed as providing a coarse approximation of the signal, with the wavelet coefficients providing higher-frequency "detail" information.

Wavelets serve as an approximate Karhunen-Loève transform for $\mathrm{fBm}$ [3], fGn, and more general LRD signals [9]. Thus, highlycorrelated, LRD signals become nearly uncorrelated in the wavelet domain. In addition, the energy of the wavelet coefficients of continuous-time $\mathrm{fBm}$ decays with scale according to a power law [3]. While for sampled $\mathrm{fBm}$ the power-law decay is not exact [3], the Haar wavelet transform of $\mathrm{fGn}$ exhibits power-law scaling of the form ${ }^{1}$ [9]

$$
\operatorname{var}\left(W_{j, k}\right)=\sigma^{2} 2^{(2 H-1)(j-1)}\left(2-2^{2 H-1}\right),
$$

where $\sigma^{2}$ is the variance of the fGn process.

\section{THE MWM}

The basic idea behind the MWM is simple. To model nonnegativity, we use the Haar wavelet transform with special wavelet-domain constraints. To capture LRD, we characterize the wavelet energy decay as a function of scale.

\subsection{Haar Wavelets and Non-Negative Data}

Before we can model non-negative signals using the wavelet transform, we must develop conditions on the scaling and wavelet coefficient values for $x(t)$ in (6) to be non-negative. While cumbersome for a general wavelet system, these conditions are simple for the Haar system. In a Haar transform (see Figure 1), the scaling and wavelet coefficients can be recursively computed using [8]

$$
\begin{aligned}
u_{j+1, k} & =2^{-1 / 2}\left(u_{j, 2 k}+u_{j, 2 k+1}\right) \\
w_{j+1, k} & =2^{-1 / 2}\left(u_{j, 2 k}-u_{j, 2 k+1}\right)
\end{aligned}
$$

\footnotetext{
${ }^{1}$ We use capital letters when we consider the underlying signal $X$ (and, hence, its wavelet and scaling coefficients) to be random.
}

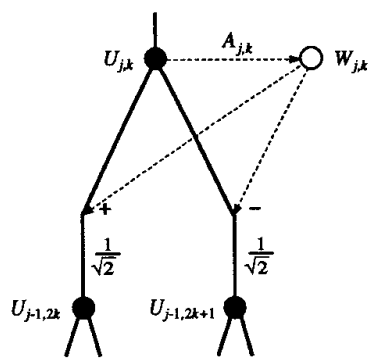

(a)

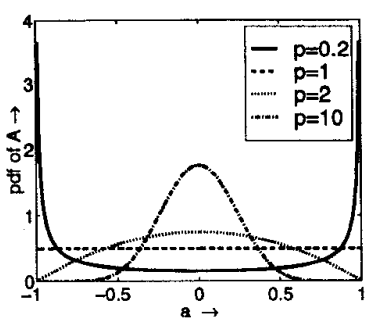

(b)
Figure 2: (a) MWM construction: At scale $j$, we form the wavelet coefficient as the product $W_{j, k}=A_{j, k} U_{j, k}$. Then, at scale $j-1$, we form the scaling coefficients $U_{j-1,2 k}$ and $U_{j-1,2 k+1}$ as sums and differences of $U_{j, k}$ and $W_{j, k}$ (normalized by $1 / \sqrt{2}$ ). (b) Probability density function of a $\beta(p, p)$ random variable $A$. For $p=0.2, A$ resembles a binomial random variable, and for $p=1$ it has a uniform density. For $p>1$ the density appears like a truncated Gaussian density, and as $p$ increases, the density resembles a Gaussian density more and more closely.

Solving (8) for $u_{j, 2 k}$ and $u_{j, 2 k+1}$ we find

$$
\begin{aligned}
u_{j, 2 k} & =2^{-1 / 2}\left(u_{j+1, k}+w_{j+1, k}\right) \\
u_{j, 2 k+1} & =2^{-1 / 2}\left(u_{j+1, k}-w_{j+1, k}\right) .
\end{aligned}
$$

For non-negative signals, $u_{j, k} \geq 0 \forall j, k$, which with (9) implies that

$$
\left|w_{j, k}\right| \leq u_{j, k}, \quad \forall j, k
$$

\subsection{Multiplicative Model}

The positivity constraints $(10)$ on the Haar wavelet coefficients lead us to a very simple multi-scale, multiplicative signal model for positive processes. (See [10] for a similar model used as an intensity prior for wavelet-based image estimation.) Let $A_{j, k}$ be a random variable supported on the interval $[-1,1]$ and define the wavelet coefficients recursively by

$$
W_{j, k}=A_{j, k} U_{j, k} .
$$

Together with (9) we obtain

$$
\begin{aligned}
U_{j, 2 k} & =2^{-1 / 2}\left(1+A_{j+1, k}\right) U_{j+1, k} \\
U_{j, 2 k+1} & =2^{-1 / 2}\left(1-A_{j+1, k}\right) U_{j+1, k} .
\end{aligned}
$$

The above construction can be visualized as a course-to-fine synthesis (see Figure 2(a)). Starting from the coarsest scale $j=$ $J_{0}$, we can synthesize a realization of a process by iteratively applying (11) to obtain the wavelet coefficients at scale $j$ and then applying (9) to obtain the scaling coefficients at the next finest scale $j-1$. When we reach the finest scale $j=J_{1}$ we obtain the desired process $X$, which is given by

$$
X[k]=2^{J_{1} / 2} U_{J_{1}, k} \text { for } k=0, \ldots, 2^{J_{0}-J_{1}}-1 .
$$

In essence the above algorithm simultaneously synthesizes the wavelet coefficients and inverts the wavelet transform, requiring only $O(N)$ operations to create a length- $N$ signal. 


\section{3. $\beta$ multipliers}

We need to choose an appropriate distribution for the multiplier $A_{j, k}$. We will assume that $A_{j, k}$ is independent of $U_{j, k}$. Second, we will assume that $A_{j, k}$ is symmetric about 0 ; it is easily shown this symmetry is necessary for the resulting process to be stationary [7].

Because of its simplicity and fiexibility, we will use a symmetric beta distribution, $\beta(p, p)$ (see Figure 2 (b)) for the $A_{j, k}$ 's and christen the resulting model the $\beta$ MWM. The variance of a random variable $A \sim \beta(p, p)$ is

$$
\operatorname{var}[A]=\frac{1}{2 p+1}
$$

\subsection{Covariance matching}

Since we assume the multipliers are identically distributed within scale (i.e., $A_{j, k} \sim \beta\left(p_{j}, p_{j}\right)$ ), for our Haar wavelet construction we can control the wavelet energy decay across scale via

$$
\frac{\operatorname{var}\left(W_{j+1, k}\right)}{\operatorname{var}\left(W_{j, k}\right)}=\frac{2 \operatorname{var}\left[A_{j+1, k}\right]}{\operatorname{var}\left[A_{j, k}\right]\left(1+\operatorname{var}\left[A_{j+1, k}\right]\right)} .
$$

Thus, to model a given process with the $\beta M W M$, we can select the parameters $p_{j}$ via (14) and (15) to match the signal's theoretical wavelet-domain energy decay, such as (7). Or, given training data, we can select the parameters to match the sample variances of the wavelet coefficients as a function of scale.

To complete the modeling, we only require to choose the coarsest scaling coefficients $U_{J_{0}, k}$ and the parameter $p_{\left(J_{0}\right)}$ of the model. From (11) and (14) we obtain

$$
\operatorname{var}\left(W_{J_{0}, k}\right) \cdot\left(2 p_{\left(J_{0}\right)}+1\right)=\mathbb{E}\left[U_{J_{0}, k}^{2}\right] .
$$

We choose a coarsest scale $J_{0}$ and obtain $p_{\left(J_{0}\right)}$ from estimates of $\mathbb{E}\left[U_{J_{0}, k}^{2}\right]$ and $\operatorname{var}\left(W_{J_{0}, k}\right)$ using (16). We then synthesize $b$ blocks of data, each with coarsest scale $J_{0}$ and finest scale $J_{1}$ (see Section 4.2) and concatenate them to obtain a synthetic data trace of $b$. $2^{J_{0}-J_{1}}$ data points. To ensure the non-negativity of our process, we would have to model $U_{J_{0}, k}(k=0, . ., b-1)$ using multivariate densities with strictly non-negative support. However, $\mathbb{E}\left[U_{J_{0}, k}\right]$ will often so greatly outweigh $\operatorname{var}\left[U_{J_{0}, k}\right]$ that the probability of a negative value will be negligible, even for a jointly Gaussian model.

Interestingly, the wavelet coefficients of the MWM are uncorrelated. This is easily deduced from the fact that the $A_{j, k}$ 's are zero mean and independent, and from the dependency of wavelet coefficients across scale

$$
W_{j, 2 k}=2^{-1 / 2} \frac{A_{j, 2 k}}{A_{j+1, k}}\left(1+A_{j+1, k}\right) W_{j+1, k}
$$

and their dependency within scale

$$
W_{j, 2 k+1}=\frac{A_{j, 2 k+1}}{A_{j, 2 k}} \frac{1-A_{j+1, k}}{1+A_{j+1, k}} W_{j, 2 k} .
$$

[7]. Thus, by matching the variances of wavelet coefficients, the MWM would exactly capture the entire correlation structure of the wavelet coefficients, if the wavelet coefficients were truly decorrelated. Typically, the correlation between the wavelet coefficients of LRD processes are small [9], and therefore we can approximate such processes quite accurately with the MWM.

Of course, this analysis addresses only the second-order statistics of our signal. Higher-order properties of the MWM are the subject of a multifractal analysis.

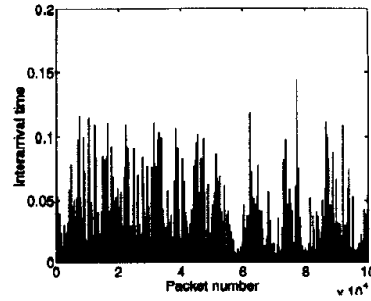

(a)

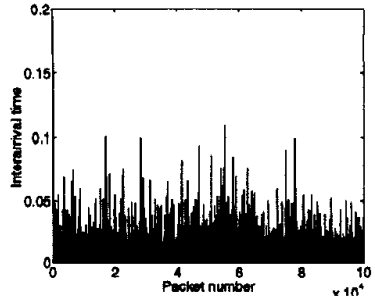

(b)
Figure 3: Inter-arrival times of packets of (a) Bellcore August 1989 [2] and (b) one realization of the beta multifractal wavelet model ( $\beta M W M$ ) synthesis. The two traces look similar at higher aggregation levels also.

\section{THE MWM IS A MULTIFRACTAL}

Multifractals offer a wealth of processes that are novel in many respects. The backbone of a multifractal is typically a construction where one starts at a coarse scale and develops details of the process on finer scales iteratively in a multiplicative fashion. It follows from (12) that the MWM is a binomial cascade, ${ }^{2}$ one of the simplest multifractals. The name binomial cascade is explained by applying (12) iteratively and writing $U_{j, k}$ as the product of the coarsest scale $U_{J_{0}, k}$ and the multipliers $2^{-1 / 2}\left(1 \pm A_{j+1, k}\right)$.

Multiplicative structures, in particular the product representation of $U_{j, k}$, bear various consequences. First, if all multipliers $1 \pm A_{j+1, k}$ in (12) are log-normal, then the marginals $U_{j, k}$ will be log-normal as well. Similarly, if the $1 \pm A_{j, k}$ are all identically distributed, $U_{j, k}$ will be approximately log-normal by the central limit theorem.

Second, interpreting $U_{j, k}$ as the increment of a limiting process $Y$ over the interval $\left[k 2^{-n},(k+1) 2^{-n}\right]$, we find for $Y$ a local behavior of the type (2). To see this, note that $\log \left|U_{j, k}\right| / \log 2^{j}$ can be written as the sum of approximately $j$ factors of the form $\log _{2} 2^{-1 / 2}\left(1 \pm A_{l, m}\right)$ normalized by $1 / j$. So, we expect this number to converge to some limiting value $H$. It is essential to note, however, that this value $H$ depends now on $t-$ hence the term multifractal for $Y$.

As a further feature of interest, depending on the moments of the multipliers, the marginals $U_{j, k}$ of binomial cascades may have diverging moments of order $q$ larger than some $q_{\text {crit }}$, where $q_{\text {crit }}$ can be arbitrarily large. This broadens the realm of "heavy tailed" processes considerably.

The exact multifractal properties of the MWM are studied in detail in [7].

\section{APPLICATION TO NETWORK TRAFFIC}

We demonstrate the power of the $\beta \mathrm{MWM}$ for a problem of considerable practical interest - network traffic modeling. The LRD of data traffic can lead to higher packet loss than that predicted by classical queuing analysis [2]. A better understanding of the LRD data traffic can thus prove invaluable in solving network problems caused by LRD. Synthetic traffic models are well-suited for this purpose, because the effect of various model parameters on network performance can be studied through simulation. The MWM is one such model because it can capture vital characteristics of real traffic like LRD and queuing behavior. To show this, we focus

\footnotetext{
${ }^{2}$ For a detailed introduction to binomial cascades and multifractal theory, see $[4,7]$.
} 


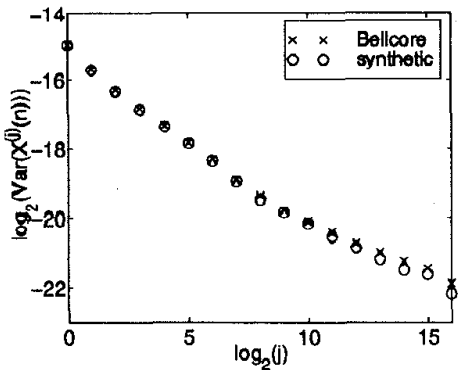

Figure 4: Variance-time plot of the Bellcore data " $x$ " and one realization of the $\beta M W M$ synthesis " 0 ".

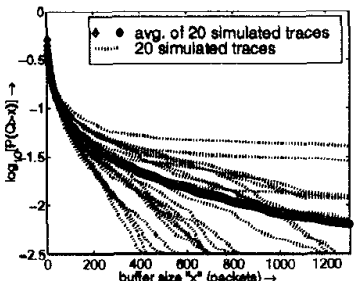

(a)

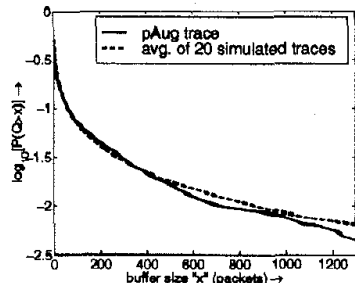

(b)
Figure 5: Comparison of the queuing behavior of the Bellcore pAug with that of 20 synthesized $\beta M W M$ traces. Displayed are the tail probabilities of buffer occupancy against buffer size. In (a), observe the variability of the queue performance of the synthesized traces. In (b), observe that the average queue performance of simulated traces and that of the real trace match closely.

on the August 1989 Bellcore Ethernet trace $p A u g$, a record of onemillion inter-arrival times (Figure 3(a)), as measured by Leland et al. [2]. From Figure 3(a), it is clear that the data has non-Gaussian marginals contradictory to the hypothesis of an $\mathrm{fBm}$ or fGn model.

We analyze the LRD properties of the trace by estimating the variance-time plot, as shown in Figure 4(a). Although the data exhibits LRD (average slope corresponding to $H=0.8$ ), the data does not appear to be strictly second-order self-similar, as evidenced by the "kink" in the slope. Again, an $\mathrm{fBm}$ or fGn model would be somewhat inaccurate.

We modeled this data using the $\beta \mathrm{MWM}$. To train the $\beta \mathrm{MWM}$, we split the Bellcore inter-arrival times into a series of $2^{16}$ length blocks, took a 16-scale Haar DWT for each block (truncating any leftover data), and calculated statistics for the scaling and wavelet coefficients. We used (14) and (15) to chose the $\beta\left(p_{j}, p_{j}\right)$ distribution used at each scale $j$ so that the theoretical variances of our synthesized wavelet coefficients matched the measured variances of the Bellcore wavelet data.

In Figures 3 and 4, we see that the synthesized data captures much of the gross structure of the Bellcore data, both in terms of marginal densities (definitely non-Gaussian) and of LRD, as evidenced through the variance-time plot. In [7], we further provide an empirical multifractal analysis of the synthesized data and show that it is closely matched by that of the Bellcore data.

To assess the accuracy and usefulness of the $\beta \mathrm{MWM}$ for traffic modeling, we compare the queuing behavior of the simulated traffic traces against that of the actual Bellcore data. In our simulation experiments, we consider the performance of an infinite length single server queue with a constant service rate of 500 packets/sec. The queuing behavior of 20 simulated traces is depicted in Figure 5(a). On comparing average queuing behavior of the 20 simulated traces with that of the real trace (Figure 5(b)), we observe that they are almost identical, ${ }^{3}$ which demonstrates the potential usefulness of the $\beta \mathrm{MWM}$ for network modeling and simulation.

\section{CONCLUSIONS}

The multiplicative wavelet model (MWM) combines the power of multifractals with the efficiency of the wavelet transform to form a flexible framework natural for characterizing and synthesizing positive-valued data with LRD. As our numerical experiments have shown, the MWM is particularly suited to the analysis and synthesis of TCP network traffic data. In addition, the model could find application in areas as diverse as financial time-series characterization, geophysics (using 2-d and 3-d wavelets), and texture modeling. The parameters of the MWM are simple enough to be easily inferred from observed data or chosen a priori. Computations involving the MWM are extremely efficient - synthesis of a trace of $N$ sample points requires only $O(N)$ computations. Finally, several extensions to the MWM are straightforward. The choice of $\beta$-distributed wavelet multipliers $A_{j, k}$ is not essential. Alternatively, we can employ mixtures of $\beta$ 's or even purcly discrete distributions to fit higher-order multifractal moments.

\section{REFERENCES}

[1] T. Lundahl, W. Ohley, S. Kay, and R. Siffert, "Fractional Brownian motion: A maximum likelihood estimator and its application to image texture," IEEE Trans. on Medical Imaging, vol. 5, pp. 152-161, Sep. 1986.

[2] W. Leland, M. Taqqu, W. Willinger, and D. Wilson, "On the selfsimilar nature of ethernet traffic (extended version)," IEEEACM Transactions on Networking, pp. 1-15, 1994.

[3] P. Flandrin, "Wavelet analysis and synthesis of fractional Brownian motion," IEEE Trans. Inform. Theory, vol. 38, pp. 910-916, Mar. 1992.

[4] R. Riedi and J. L. Véhel, "TCP traffic is multifractal: a numerical study," IEEE Trans. Networking, submitted Oct. 1997. Available at www.dsp.rice.edu.

[5] J. L. Vêhel and R. Riedi, "Fractional Brownian motion and data traffic modeling: The other end of the spectrum," Fractals in Engineering, pp. 185-202, 1997.

[6] B. B. Mandelbrot, "Intermittent turbulence in self similar cascades : divergence of high moments and dimension of the carrier," J. Fluid. Mech, vol. 62, p. 331, 1974.

[7] R. H. Riedi, M. S. Crouse, V. Ribeiro, and R. G.Baraniuk, "A multifractal wavelet model with application to TCP network traffic," Submitted to IEEE Trans. on Info. Theory, 1998. Available at www.dsp.rice.edu.

[8] I. Daubechies, Ten Lectures on Wavelets. New York: SIAM, 1992.

[9] L. M. Kaplan and C.-C. J. Kuo, 'Extending self-similarity for fractional Brownian motion," IEEE Trans. on Signal Proc., vol. 42. pp. 3526-3530, Dec. 1994.

[10] K. E. Timmerman and R. D. Nowak, "Multiscale Bayesian estimation of Poisson intensities," in Proc. 31st Asilomar Conf., (Pacific Grove, CA), Nov. 1997.

\footnotetext{
${ }^{3}$ A real trace can be assumed to be a single realization of our model and so its queuing performance cannot always be expected to closely match that of the average of several simulated traces (see [7] for more queuing experiments).
} 